\title{
Charged pions tagged with polarized photons probing strong CP violation in a chiral-imbalance medium
}

\author{
Mamiya Kawaguchi*, ${ }^{1}$ Masayasu Harada ${ }^{\dagger}{ }^{1}$ Shinya Matsuzaki ${ }^{\ddagger},{ }^{1,2}$ and Ruiwen Ouyang ${ }^{\S 3}$ \\ 1 Department of Physics, Nagoya University, Nagoya 464-8602, Japan. \\ 2 Institute for Advanced Research, Nagoya University, Nagoya 464-8602, Japan. \\ 3 College of Physics, Jilin University, Changchun, 130012, China
}

(Dated: June 13, 2017)

\begin{abstract}
It is expected that in a hot QCD system, a local parity-odd domain can be produced due to nonzero chirality, which is induced from the difference of winding numbers carried by the gluon topological configuration (QCD sphaleron). This local domain is called the chiral-imbalance medium characterized by nonzero chiral chemical potential, which can be interpreted as the time variation of the strong $\mathrm{CP}$ phase. We find that the chiral chemical potential generates the parity breaking term in the electromagnetic form factor of charged pions. Heavy ion collision experiments could observe the phenomenological consequence of this parity-odd form factor through the elastic scattering of a pion and a photon in the medium. Then we quantify the asymmetry rate of the parity violation by measuring the polarization of the photon associated with the pion, and discuss how it could be measured in a definite laboratory frame. We roughly estimate the typical size of the asymmetry, just by picking up the pion resonant process, and find that the signal can be sufficiently larger than possible background events from parity-breaking electroweak process. Our findings might provide a novel possibility to make a manifest detection for the remnant of the strong $\mathrm{CP}$ violation.
\end{abstract}

\section{INTRODUCTION}

It is still mysterious whether or not the $\mathrm{CP}$ violation by strong interactions of QCD (strong CP violation) certainly exists and one could observe a finite size of its violation. To reveal the mystery, many theoretical and experimental challenges (such as detecting the electric dipole moment of neutron) have so far been met. However, the strong CP violation has not yet been detected because of its extremely tiny size.

The strong CP phase arises from the axial anomaly by converting the difference of the winding numbers $\left(\Delta N_{W}\right)$, which is transferred by a topologically-nontrivial gluon configuration into the nonzero chirality $\left(\Delta N_{L-R}\right)$; $\left(\Delta N_{W} \rightarrow \Delta N_{L-R}\right)$. The difference of the winding numbers $\left(\Delta N_{W}\right)$ is created by the transition between two of the infinite number of vacua forming the $\theta$ vacuum. In this sense, the detectability of the strong $\mathrm{CP}$ violation could depend on the survival probability of the transition rate with nonzero $\Delta N_{W}$. This transition rate is, however, known to be extremely smaller than the QCD time scale, which might thus cause difficulty in detecting the strong $\mathrm{CP}$ violation.

To access such detectability of the strong CP phase, it would be intriguing to place QCD in hot and/or dense environments. Because of the existence of the thermally excited-topologically nontrivial gauge configuration (the so-called sphaleron [1, 2]), in contrast to the vacuum case

\footnotetext{
*mkawaguchi@hken.phys.nagoya-u.ac.jp

†harada@hken.phys.nagoya-u.ac.jp

‡synya@hken.phys.nagoya-u.ac.jp

§ ruiwen.ouyang@gmail.com
}

as above, the difference of winding numbers $\left(\Delta N_{W}\right)$ can survive within the QCD time scale [3 6]. When (almost massless) quarks couple to such a topological gauge configuration, the chirality imbalance $\left(\Delta N_{L-R}\right)$ as well as the strong CP phase would be present in the hot QCD system. The strong CP violation along with the chiral imbalance thus would (locally) be measured within the QCD time scale, due to the existence of the sphaleron configuration [3 6 ].

The existence of the chirality imbalance would imply the presence of a "chemical potential", the so-called chiral chemical potential $\mu_{5}$, which acts as a constant all over the hot QCD system and gives the energy difference $\left(\Delta \mathcal{E}_{L-R}\right)$ between chiral quarks, such as $\Delta \mathcal{E}_{L-R}=$ $-\mu_{5} \Delta N_{L-R}$ [3]. Such a local domain would therefore look like a medium, called the chiral-imbalance medium, where the preference of chiralities (such as $\Delta \mathcal{E}_{L-R}$ ) and the $\mathrm{CP}$ violation are locally present.

Heavy-ion collision experiments might form such metastable CP-odd domains characterized by the chiral chemical potential $\mu_{5}$, as has so far been addressed in the literature, e.g., [7-10] and [11, 12].

In this paper, we discuss a novel possibility to observe the strong $\mathrm{CP}$ violation in the chiral-imbalance medium: the new probe might be a polarized photon tagged with a charged pion coming out of the chiral-imbalance medium created in heavy ion collisions.

\section{THE CHIRAL IMBALANCE MEDIUM}

We begin with a brief review of the theoretical and phenomenological grounds. In heavy ion collision experiments, the hot QCD medium (the so-called fireball) is 
created at the time scale $\tau_{\text {fireball }}$ of $\mathcal{O}(1-10)$ fm after the collision (for a recent review, see [13]). As discussed in the literature [8 12], we may take the $\theta$ parameter to be position-time dependent, i.e., $\theta=\theta(x)$ : the $\theta$ fluctuation in time will be interpreted as the chiral chemical potential $\mu_{5}$, as will be seen below. The QCD $\theta$ term then looks like

$$
\int_{\mathrm{bulk}} d^{4} x \theta(x) \cdot \frac{g_{s}^{2}}{32 \pi^{2}} \epsilon^{\mu \nu \rho \sigma} \operatorname{tr}\left[G_{\mu \nu} G_{\rho \sigma}\right],
$$

where the symbol "bulk" denotes the volume of the fireball bulk and $G_{\mu \nu}$ is the gluon-field strength, $G_{\mu \nu}=$ $\partial_{\mu} G_{\nu}-\partial_{\nu} G_{\mu}-i g_{s}\left[G_{\mu}, G_{\nu}\right]$ with the QCD gauge coupling $g_{s}$.

One should recall that the $\theta$ term is related to the axial-anomaly form for the isosinglet axial current $j_{5, \mu}$,

$$
\partial^{\mu} j_{5, \mu}=\sum_{f} 2 m_{f} \bar{q}_{f} i \gamma_{5} q_{f}-\frac{g_{s}^{2} N_{f}}{16 \pi^{2}} \epsilon^{\mu \nu \rho \sigma} \operatorname{tr}\left[G_{\mu \nu} G_{\rho \sigma}\right]
$$

where $q_{f}$ denotes the quark field, $m_{f}$ is the quark mass, and $N_{f}$ stands for the number of the quark flavors. Using Eq.(2), one finds that Eq.(1) goes like

$$
\int_{\mathrm{bulk}} d^{4} x\left(\frac{\partial_{\mu} \theta(x)}{2 N_{f}}\right) \cdot j_{5}^{\mu}(x),
$$

(up to the terms of the form $\theta \cdot m_{f}$ times pseudoscalar density) where integration by parts has been done. Looking at Eq.(3), we thus readily find that the time variation of $\theta$ plays the role of the chiral chemical potential [8 $[12]$ :

$$
\begin{aligned}
& \left(\frac{\partial_{0} \theta(x)}{2 N_{f}}\right) \equiv \mu_{5} \\
\text { s.t. } & \int_{\text {bulk }} d t \mu_{5} \cdot Q_{5}(t)=-\mu_{5} \Delta N_{L-R},
\end{aligned}
$$

where $\Delta N_{L-R}=-\int d t Q_{5}(t)=-\int d^{4} x j_{5}^{0}(x)$. In that case the chiral chemical potential is embedded into the time component of the axialvector source $\mathcal{A}_{\mu}^{0}$ as [10]

$$
\mathcal{A}_{\mu}^{0}=\left(\mu_{5}, \overrightarrow{0}\right)^{T} \text {. }
$$

Through the axialvector source coupled to the quark chiral current, we are thus able to address hadron physics in the chiral-imbalance medium, where the size of $\mu_{5}$ is expected to be on the order of QCD scale, $\mathcal{O}(100 \mathrm{MeV})$.

In the presence of the chiral chemical potential $\mu_{5}$, the parity symmetry as well as the Lorentz symmetry are broken, and one is then left with the three-dimensional $S O(3)$ symmetry. This parity breaking effect would be transferred by hadrons, for instance, through their interactions with external gauge currents, arising from the intrinsic-parity odd form like the $\pi^{0} \gamma \gamma$ vertex. (Here the intrinsic parity is defined to be even when a particle has the parity $(-1)^{\text {spin }}$, otherwise it is odd.) Several studies regarding this kind of parity breaking effects have so far been done in the context of hadron physics under a strong field configuration (e.g., [14, 15]), or under the influence of the nonzero chiral chemical potential without strong fields [11, 12].

\section{PION FORM FACTORS IN THE CHIRAL IMBALANCE MEDIUM}

We propose a new parity violating process related to the pion-electromagnetic form factors arising from intrinsic parity-odd interactions in the chiral imbalance medium. We derive its phenomenological consequence to discuss a new detection possibility for the strong CP violation in heavy ion collision experiments.

We first consider the general expression for the form factors of the charged pion coupled to the background photon field $A^{\mu}=\left(A^{0}, A^{i}=\vec{A}\right)(i=1,2,3)$ in the presence of parity violation and $S O(3)$ symmetry. It is written as

$$
\begin{aligned}
& \left\langle\pi^{+}\left(p^{\prime}=q+p\right)\left|\mathcal{J}^{\mu}(0)\right| \pi^{+}(p)\right\rangle \\
= & e F_{1} \cdot\left(p^{0}+p^{\prime 0}\right) \delta_{0}^{\mu}+e F_{2} \cdot\left(p^{i}+p^{i}\right) \delta_{i}^{\mu} \\
& +e F_{3} \cdot\left(q^{i}\right) \delta_{i}^{\mu}+i e F_{4} \cdot \epsilon^{0 \mu \nu \rho} p_{\nu} p_{\rho}^{\prime},
\end{aligned}
$$

where $q$ denotes the transfer momentum $q ; \mathcal{J}^{\mu}$ is the electromagnetic current (including nonminimal forms in general); $e$ is the electromagnetic coupling and $F_{i=1,2,3,4}=$ $F_{i=1,2,3,4}\left(q^{0}, p^{0},|\vec{q}|,|\vec{p}|\right)$ denote the form factors. In Eq.(6), just for later convenience, we have defined the form factor $F_{1}$ by extracting the factor $\left(p^{0}+p^{\prime 0}\right)$. In terms of the effective action, the first term and second term correspond to the $\pi^{+}-\pi^{-}-\gamma$ interactions such as $A^{0} j^{0}$ and $\vec{A} \cdot \vec{j}$, respectively, where $j^{\mu}$ is the pion electromagnetic current of the minimal coupling form: $j_{\mu}=i e\left(\partial_{\mu} \pi^{+} \cdot \pi^{-}-\partial_{\mu} \pi^{-} \cdot \pi^{+}\right)$. The third term can be absorbed into a redefinition of the other form factors by imposing the Ward-Takahashi identity for the electromagnetic gauge invariance. The fourth term $F_{4}$ is the one that will be induced in the presence of $\mu_{5}$ in the chiral-imbalance medium, as will be shown below $\# 1$.

To see how the $F_{4}$ form factor shows up in the chiralimbalance medium with nonzero $\mu_{5}$, we employ the chiral Lagrangian based on the coset space $G / H=$ $\left[U(2)_{L} \times U(2)_{R}\right] / U(2)_{V}$ and the Wess-Zumino-Witten (WZW) term [17]:

$$
S=\int d^{4} x \frac{f_{\pi}^{2}}{4} \operatorname{tr}\left[D_{\mu} U^{\dagger} D^{\mu} U\right]+S_{\mathrm{WZW}},
$$

where $f_{\pi}(\simeq 92 \mathrm{MeV})$ is the pion decay constant, and $U$ denotes the chiral field parameterizing the pion fields as $U=\exp \left(\sum_{a=1}^{3} 2 i \pi^{a}\left(\sigma^{a} / 2\right) / f_{\pi}\right)$ with $\sigma^{a}$ being the Pauli matrices. Here we simply ignored the $U(1)_{A}$ pion component because it is irrelevant to the present our proposal. The covariant derivative acting on $U$ is defined as $D_{\mu} U=\partial_{\mu} U-i \mathcal{L}_{\mu} U+i U \mathcal{R}_{\mu}$ with the external gauge fields

\#1 The $F_{4}$ type form factor would also be induced when one employs some dense matter system with other chiral chemical potentials including the isospin breaking effect (called the chiralisospin chemical potential; e.g., see [16]). 
$\mathcal{L}_{\mu}$ and $\mathcal{R}_{\mu}$ arising by gauging the chiral $U(2)_{L} \times U(2)_{R}$ symmetry. The photon field and the chiral chemical potential $\mu_{5}$ are then introduced as a part of the external vector and axialvector gauge fields $\left(\mathcal{V}_{\mu}, \mathcal{A}_{\mu}\right)$ as

$$
\begin{aligned}
& \mathcal{V}_{\mu}=\frac{1}{2}\left(\mathcal{R}_{\mu}+\mathcal{L}_{\mu}\right)=e Q_{\mathrm{em}} A_{\mu}, \\
& \mathcal{A}_{\mu}=\frac{1}{2}\left(\mathcal{R}_{\mu}-\mathcal{L}_{\mu}\right)=\mu_{5} \delta_{\mu 0} \cdot 1_{2 \times 2},
\end{aligned}
$$

where the electromagnetic charge matrix is defined as $Q_{\mathrm{em}}=\sigma_{3} / 2+1_{2 \times 2} / 6$.

The WZW term in Eq. (7) includes the following interactions between the pion and external gauge fields:

$$
\begin{aligned}
S_{\mathrm{WZW}}= & i \frac{N_{c}}{48 \pi^{2}} \int d^{4} x \epsilon^{\mu \nu \rho \sigma} \operatorname{tr}\left[\left\{\partial_{\mu} \mathcal{L}_{\nu}, \mathcal{L}_{\rho}\right\}\left(\partial_{\sigma} U\right) U^{\dagger}\right. \\
& +\left\{\partial_{\mu} \mathcal{R}_{\nu}, \mathcal{R}_{\rho}\right\} U^{\dagger} \partial_{\sigma} U-\partial_{\mu} \mathcal{L}_{\nu} \partial_{\rho} U \mathcal{R}_{\sigma} U^{\dagger} \\
& +\partial_{\mu} \mathcal{R}_{\nu} \partial_{\rho} U^{\dagger} \mathcal{L}_{\sigma} U-\mathcal{R}_{\mu} U^{\dagger} \mathcal{L}_{\nu}\left(\partial_{\rho} U\right) U^{\dagger} \partial_{\sigma} U \\
& +U^{\dagger} \mathcal{L}_{\mu} U \mathcal{R}_{\nu} U^{\dagger}\left(\partial_{\rho} U\right) U^{\dagger}\left(\partial_{\sigma} U\right) \\
& -\frac{1}{2} \mathcal{L}_{\mu}\left(\partial_{\nu} U\right) U^{\dagger} \mathcal{L}_{\rho}\left(\partial_{\sigma} U\right) U^{\dagger} \\
& \left.+\frac{1}{2} \mathcal{R}_{\mu}\left(\partial_{\nu} U\right) U^{\dagger} \mathcal{R}_{\rho}\left(\partial_{\sigma} U\right) U^{\dagger}\right]+\cdots
\end{aligned}
$$

with the number of colors $N_{c}=3$. Thus, we extract the electromagnetic interactions of the charged pion in the chiral-imbalance medium including the WZW term to get

$$
D_{\mu} \pi^{+} D^{\mu} \pi^{-}+\frac{i e \mu_{5} N_{c}}{6 \pi^{2} f_{\pi}^{2}} \epsilon^{0 \nu \rho \sigma} \partial_{\nu} \pi^{+} \partial_{\rho} \pi^{-} A_{\sigma},
$$

where $D_{\mu} \pi^{ \pm}=\left(\partial_{\mu} \mp i e A_{\mu}\right) \pi^{ \pm}$. Obviously, the second term breaks the parity, but preserves the charge conjugation, which indicates the $\mathrm{CP}$ violation with nonzero $\mu_{5}$. From Eq. (10) we read off the electromagnetic form factors corresponding to the general expressions in Eq.(6):

$$
\begin{aligned}
& F_{1}=F_{2}=1, \\
& F_{3}=0, \\
& F_{4}=\frac{\mu_{5} N_{c}}{6 \pi^{2} f_{\pi}^{2}} .
\end{aligned}
$$

The result on $F_{1}=1$ in Eq.(11) just tells us that the pion electromagnetic charge is correctly normalized, which just reflects the electromagnetic gauge invariance, while the normalization for $F_{2}=1$ has happened by accident. It is interesting to note that, although the pion is manifestly the spin 0 particle, the nonzero $F_{4}$ term looks like the anomalous magnetic moment of the charged pion like $\vec{B} \cdot \vec{j}$, when the charged pions are set in an orientedmagnetic field background.

\section{MEASURING PARITY VIOLATION IN THE PION-PHOTON SCATTERING}

Now we propose a new possibility to detect the remnant of the strong $\mathrm{CP}$ violation mimicked by nonzero chiral chemical potential $\mu_{5}$ involved in the $F_{4}$ form factor.
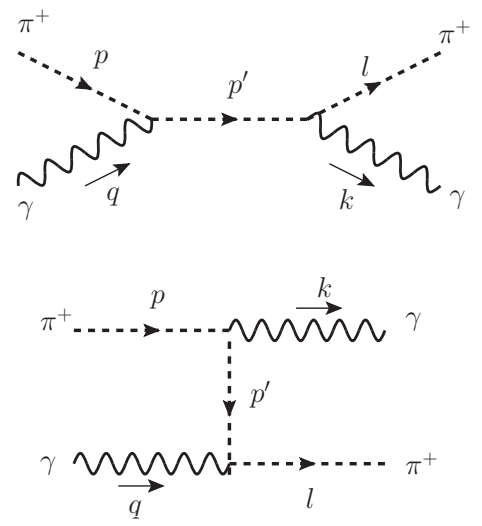

FIG. 1: The Feynman graphs illustrating $\pi^{ \pm} \gamma \rightarrow \pi^{ \pm} \gamma$ scattering process involving the parity breaking form factor $F_{4}$ and the parity conserving $F_{1}\left(=F_{2}\right)$ in Eq. (11).

The $\mu_{5}$-dependent $F_{4}$ generates $\pi^{ \pm} \gamma \rightarrow \pi^{ \pm} \gamma$ scattering processes as depicted in Fig. 1 ${ }^{\# 2}$. In light of the heavy ion collisions, the charged pion and photon in the initial state (corresponding to the left-side states for each graph in Fig. (1) come from the chiral-imbalance medium, so we consider the unpolarized photon with the sum and average over spin properly taken. In the final state (depicted in the right-side states for each graph in Fig. 10 we impose that the photon be polarized with certain spin $(s)$ so as to make the helicity dependence $(\lambda \propto \vec{k} \cdot \vec{s})$ with respect to the photon outgoing momentum $(\vec{k})$. Thus the following two processes are parity conjugate:

$$
\begin{aligned}
\pi^{ \pm}(\vec{p})+\gamma(\vec{q}) & \rightarrow \pi^{ \pm}(\vec{l})+\gamma_{+}(\vec{k}), \\
\pi^{ \pm}(-\vec{p})+\gamma(-\vec{q}) & \rightarrow \pi^{ \pm}(-\vec{l})+\gamma_{-}(-\vec{k}),
\end{aligned}
$$

where \pm attached on photons in the final state denote the photon helicities.

The geometrical configuration relevant to the processes in Eq. (12) in heavy ion collision experiments is sketched in Fig. 22 In the laboratory frame (defined at the rest frame of the observer/detector), we measure charged pions and photons emitted from the fireball bulk, where the photons are specified by the zenith angle $\alpha$ and the azimuthal angle $\beta$ in the laboratory coordinate space $\left(x_{\text {lab }}, y_{\mathrm{lab}}, z_{\mathrm{lab}}\right)$. Then the location of the charged pion is determined by the opening angle $\theta$ from the photon and another azimuthal angle $\phi$ on the plane transverse to the photon direction, which we shall call the "transverse plane" (see Fig. (2). The angles $\theta$ and $\phi$ correspond to kinematical variables in the final state in computing the $\pi^{ \pm} \gamma$ scattering amplitudes. As the parity conjugate

\#2 Actually, one needs another contribution arising from a contact $\pi^{ \pm} \gamma \pi^{\mp} \gamma$ vertex present in Eq. 17], to make the scattering amplitude gauge invariant. 


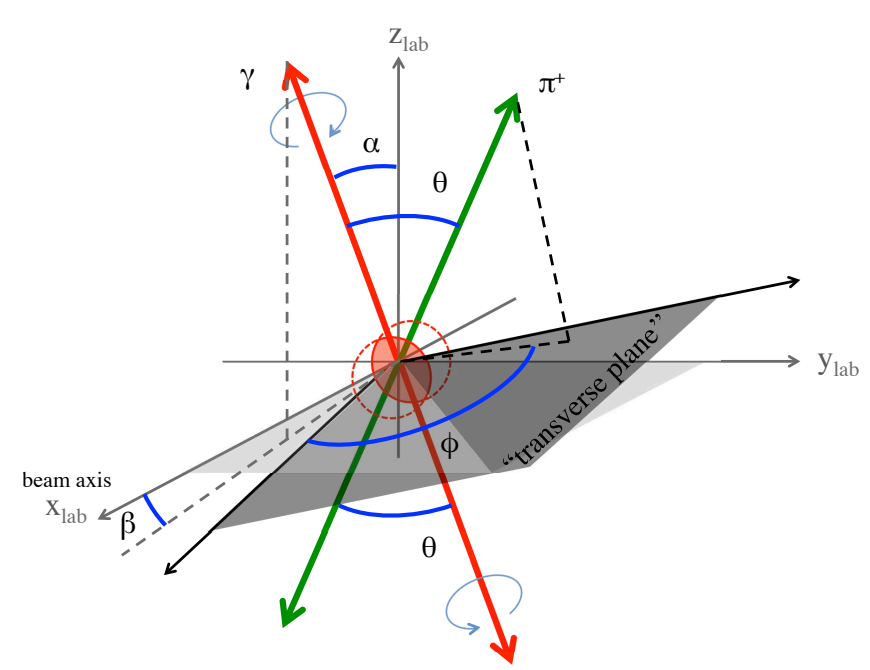

FIG. 2: A schematic sketch of the laboratory frame geometry relevant to the proposed parity violation measurement in heavy ion collisions. The laboratory frame is spanned by the coordinates $\left(x_{\mathrm{lab}}, y_{\mathrm{lab}}, z_{\mathrm{lab}}\right)$. The red blob at the origin denotes the fireball domain created by the collision of heavy ions (red dashed circles). The plane drawn in blue is set to be transverse to the photon direction, which we call the "transverse plane". The circles with arrows surrounding around the photon profile (red allows) represent the helicities. The pion trajectories (displayed as green profiles) are specified by the azimuthal angle $\phi$ measured in the "transverse plane" and the zenith angle $\theta$ with respect to the photon profiles determined by the angles $\alpha$ and $\beta$ in the laboratory frame.

event, we also observe the mirror process at the laboratory frame position with the same opening angle $\theta$ as well as the azimuthal angle $\phi$ between the charged pion and photon.

We shall suppose that those photon polarizations could be observed, say, by the circular polarization apparatus designated in the laboratory frame, with the angles $\alpha$ and $\beta$ taken into account. In that case, the number of these mirror events could be different due to the parity violation induced by the $\mu_{5}$-dependent $F_{4}$ term. Such an asymmetry $(\mathcal{A})$ can be evaluated as

$$
\mathcal{A}=\left|\frac{\mathcal{N}_{+}-\mathcal{P}\left[\mathcal{N}_{+}\right]}{\sum_{\lambda}\left\{\mathcal{N}_{\lambda}+\mathcal{P}\left[\mathcal{N}_{\lambda}\right]\right\}}\right|,
$$

where $\mathcal{N}_{\lambda}$ stands for the number of events per the phase space, $d E_{\gamma} d \cos \theta d \phi$, for the parity conjugate processes in Eq.(12) with the helicity $\lambda$ and the photon energy $E_{\gamma}$ in the final state. The symbol $\mathcal{P}$ acts as the parity conjugation projection. The denominator represents the total number of the $\pi^{ \pm} \gamma$ emission events with unpolarized photons per the phase space.

One may notice that the asymmetry in Eq.(13) generically depends on the kinematical variables associated with the initial state particles in the medium. Since the chiral imbalance medium is formed in the hot QCD bulk, it would be reasonable to take the thermal average over the phase space for the initial state, which would be subject to the strong dependence on modeling of the hot QCD medium.

Instead of the detailed computation including the thermal effects and full channel analysis contributing to the $\pi^{ \pm} \gamma$ scattering processes, here we may just estimate the typical size of the asymmetry, by focusing only on the pion resonant channel (the upper graph in Fig. 1), to get a rough implication of our proposal. In this case, the asymmetry would be free from kinematical configuration in the initial state and only depend on the final state variables $\left(\theta, \phi, E_{\gamma}\right.$ and the pion energy $\left.E_{\pi}\right)$, and hence we are allowed to express the asymmetry in Eq.(13) as

$$
\begin{aligned}
& \mathcal{A}^{\text {s-channel }}\left(E_{\gamma}, E_{\pi}, \theta, \phi\right)= \\
& \left|\frac{\mathcal{N}_{+}\left(E_{\pi}, E_{\gamma}, \theta, \phi\right)-\mathcal{P}\left[\mathcal{N}_{+}\left(E_{\pi}, E_{\gamma}, \theta, \phi\right)\right]}{\sum_{\lambda}\left\{\mathcal{N}_{\lambda}\left(E_{\pi}, E_{\gamma}, \theta, \phi\right)+\mathcal{P}\left[\mathcal{N}_{\lambda}\left(E_{\pi}, E_{\gamma}, \theta, \phi\right)\right]\right\}}\right| .
\end{aligned}
$$

Using Eq.(14) we evaluate the typical size of the asymmetry by choosing the circular polarization state for the photon in the final state. Note that the asymmetry arises from the $\mu_{5}$ term along with the loop suppression factor as

$$
\frac{N_{c} \mu_{5} E_{\pi / \gamma}}{\left(4 \pi f_{\pi}\right)^{2}} \sim \frac{\mu_{5}}{\left(4 \pi f_{\pi}\right)}=\mathcal{O}\left(10^{-1}\right),
$$

with $E_{\pi / \gamma}=\mathcal{O}\left(4 \pi f_{\pi}\right)=\mathcal{O}(1) \mathrm{GeV}$ and $\mu_{5}=\mathcal{O}(100)$ $\mathrm{MeV}$ being assumed. We may therefore expand terms in Eq. (14) in powers of that loop factor and keep only the nontrivial leading order contributions to get

$$
\begin{aligned}
& \mathcal{A}^{\mathrm{s}-\text { channel }}= \\
& \frac{\mu_{5} N_{c}}{12 \pi^{2} f_{\pi}^{2}} \frac{E_{\gamma}\left(E_{\pi}^{2}-m_{\pi}^{2}\right) \sin ^{2} \theta}{m_{\pi}^{2}+E_{\gamma} E_{\pi}\left(1-\sqrt{1-\frac{m_{\pi}^{2}}{E_{\pi}^{2}}} \cos \theta\right)} .
\end{aligned}
$$

In the limit where $E_{\pi / \gamma} \gg m_{\pi}$ and $\theta \rightarrow 0$, we find that the asymmetry reaches the maximum:

$$
\begin{aligned}
\left.\mathcal{A}^{\mathrm{s}-\text { channel }}\right|_{\max } & =\frac{\mu_{5} E_{\pi} N_{c}}{6 \pi^{2} f_{\pi}^{2}} \\
& \simeq 0.2 \times\left(\frac{\mu_{5}}{200 \mathrm{MeV}}\right)\left(\frac{E_{\pi}}{1 \mathrm{GeV}}\right)
\end{aligned}
$$

The estimated size of the asymmetry is indeed on the order of the loop suppression factor $\left(\mu_{5} /\left(4 \pi f_{\pi}\right)\right)(=$ $\left.\mathcal{O}\left(10^{-1}\right)\right)$ as we expected.

Possible background events would come from the electroweak interactions breaking the parity, for instance, from the $W$ boson exchange process in the vacuum through the $W-\pi-\gamma$ vertex arising in the WZW term. As long as one focuses on the pion pole part as evaluated in the above, however, such a $\mathrm{W}$ boson contribution would negligibly be small compared with the pion exchange term, roughly by a factor of $\mathcal{O}\left(G_{F}^{2} f_{\pi}^{4}\right)=\mathcal{O}\left(10^{-14}\right)$, where $G_{F}$ is the Fermi constant $\simeq(290 \mathrm{GeV})^{-2}$ and the typical order of all the transferred momenta and $\mu_{5}$ are set 
to $f_{\pi}$. Thus the proposed asymmetry arises dominantly from the parity violation by $\mu_{5} \neq 0$, namely, the strong $\mathrm{CP}$ violation, which could be testable in heavy ion collisions with high statistics.

\section{CONCLUSION}

In this paper, we have discussed a possibility for charged pions to act as a probe for measuring the strong $\mathrm{CP}$ violation: the charged-pion along with the photon could pick up the remnant of the $\mathrm{CP}$ violation in the chiral-imbalance medium, which can be produced in heavy ion collisions. Our proposal presented here could be tested through the parity violation in a lab frame (defined in the text), by measuring the polarized photons tagged by charged pions with finite opening angle in the lab frame of heavy ion collision experiments.

We have roughly estimated the typical size of the parity violation, quantified as the asymmetry, by focusing only on the pion exchange contribution in the so-called s-channel and find that the signal is much larger than the size of expected background from electroweak process. Inclusion of other channel contributions would not significantly make a difference from our estimate as far as the order of magnitude is concerned.

One also notices that our estimate was made by assuming the energy of charged pion to be much larger than the pion mass $\left(E_{\pi}=\mathcal{O}(1) \mathrm{GeV}\right)$. One may therefore naively suspect that other higher resonances such as the rho meson would not be negligible. However, it turns out that such higher resonance $(R)$ contributions are necessarily suppressed by a factor of $\left(m_{\pi}^{2} / m_{R}^{2}\right) \ll 1$ in the asymmetry, when the nearly collinear emission $(\theta \simeq 0)$ is selected, as done in Eq.(17): the propagator of the resonance $R$ with mass $m_{R}\left(\gg m_{\pi}\right)$ in the s-channel takes the form in the laboratory frame $\sim\left[E_{\pi} E_{\gamma}\left(1-\sqrt{1-m_{\pi}^{2} / E_{\pi}^{2}} \cos \theta\right)-\left(m_{R}^{2}-m_{\pi}^{2}\right)\right]^{-1}$. When the charged pion is emitted almost collinear to the photon (i.e. $\theta \simeq 0$ ) with the high energies $E_{\gamma / \pi} \gg m_{\pi}$, the $R$-propagator goes like $\sim m_{R}^{-2}$. Thus the estimated size of the asymmetry in Eq. (17) will be reliable even for the high energy emission of charged pions.

Note that our parity violation induced by nonzero chiral chemical potential can be seen in the laboratory frame, but cannot be seen in the resonance-mass distribution in the usual center of mass frame, such as in the dilepton mass distribution, as discussed in the literature [11]. In this sense, our findings presented in this paper certainly include a new proposal to probe the strong $\mathrm{CP}$ violation in a hot QCD medium, and could give a large enough signal in a realistic experimental situation for heavy ion collisions.

More precise estimates of the charged pion-photon scattering amplitudes including the thermal effect in the medium and the chiral-imbalance effects involving other light hadrons will be studied elsewhere, which would make our prediction more definite.

In addition to the theoretical uncertainties, however, one needs to more seriously take into account the experimental setup in heavy ion collisions. Since thousands of particles are produced in heavy-ion collisions, the analysis of specifically selected processes, such as elastic scattering of pions and photons proposed in the present paper, would be badly contaminated by the background effects; thermal emission, inelastic scattering, and so forth. Due to those complex experimental configurations, the energy range for the polarization asymmetry would need in particular to be limited to relatively low photon energy ranges, where the background would still get too large to be simply understood. Though being the definite signal for the strong $\mathrm{CP}$ violation, the presently proposed parity violation measurement would therefore be very challenging and would require high statistics and resolution, e.g., for the photon energy.

In closing, we shall give some comments on other possible phenomenological implications derived from the $\mu_{5^{-}}$ dependent form factor.

Besides the parity violation as noted above, actually, our $\mu_{5}$-induced form factor $F_{4}$ would give rise to other heavy-ion collision signatures, much like the so-called the chiral magnetic effect [8 10], which can be seen if an oriented-magnetic field is present in the fireball bulk. In particular, the $F_{4}$ form factor in Eq.(11) generates the $\vec{B} \cdot \vec{j}$ in the magnetic field background, hence the $F_{4}$ contribution would contaminate the event-by-event charge separation measurements signaling the chiral magnetic effect [18, 19]. In addition, our direct photon without the specified polarization could also mix with the signal generated from the chiral magnetic effect as discussed in [14].

\section{Acknowledgments}

We are grateful to Kenji Fukushima and Kazunori Itakura for useful comments. This work was supported in part by the JSPS Grant-in-Aid for Young Scientists (B) No. $15 \mathrm{~K} 17645$ (S.M.), and the JSPS Grant-in-Aid for Scientific Research (C) No. 16K05345 (M.H.). R.O. was partially supported by the TAQ honor program in physics from the Office of Undergraduate Education and College of Physics in Jilin University.
[1] N. S. Manton, Phys. Rev. D 28, 2019 (1983). doi:10.1103/PhysRevD.28.2019.
[2] F. R. Klinkhamer and N. S. Manton, Phys. Rev. D 30, 2212 (1984). doi:10.1103/PhysRevD.30.2212. 
[3] L. D. McLerran, E. Mottola and M. E. Shaposhnikov, Phys. Rev. D 43, 2027 (1991). doi:10.1103/PhysRevD.43.2027.

[4] G. D. Moore, Phys. Lett. B 412, 359 (1997) doi:10.1016/S0370-2693(97)01046-0.

[5] G. D. Moore and K. Rummukainen, Phys. Rev. D 61, 105008 (2000) doi:10.1103/PhysRevD.61.105008.

[6] D. Bodeker, G. D. Moore and K. Rummukainen, Phys. Rev. D 61, 056003 (2000) doi:10.1103/PhysRevD.61.056003.

[7] D. Kharzeev, A. Krasnitz and R. Venugopalan, Phys. Lett. B 545, 298 (2002) doi:10.1016/S03702693(02)02630-8 hep-ph/0109253.

[8] D. Kharzeev and A. Zhitnitsky, Nucl. Phys. A 797, 67 (2007) doi:10.1016/j.nuclphysa.2007.10.001.

[9] D. E. Kharzeev, L. D. McLerran and H. J. Warringa, Nucl. Phys. A 803, $227 \quad$ (2008) doi:10.1016/j.nuclphysa.2008.02.298.

[10] K. Fukushima, D. E. Kharzeev and H. J. Warringa, Phys. Rev. D 78, $074033 \quad$ (2008) doi:10.1103/PhysRevD.78.074033.

[11] A. A. Andrianov, V. A. Andrianov, D. Espriu and X. Planells, Phys. Lett. B 710, 230 (2012) doi:10.1016/j.physletb.2012.02.072.

[12] A. A. Andrianov, D. Espriu and X. Planells, Eur. Phys.
J. C 73, no. 1, 2294 (2013) doi:10.1140/epjc/s10052-0132294-0.

[13] R. Derradi de Souza, T. Koide and T. Kodama, Prog. Part. Nucl. Phys. 86, 35 (2016) doi:10.1016/j.ppnp.2015.09.002 arXiv:1506.03863 [nucl-th]].

[14] K. Fukushima and K. Mameda, Phys. Rev. D 86, 071501 (2012) doi:10.1103/PhysRevD.86.071501.

[15] G. Cao and X. G. Huang, Phys. Lett. B 757, 1 (2016) doi:10.1016/j.physletb.2016.03.066 arXiv:1509.06222 [hep-ph]].

[16] D. Ebert, T. G. Khunjua and K. G. Klimenko, Phys. Rev. D 94, no. 11, 116016 (2016) doi:10.1103/PhysRevD.94.116016 arXiv:1608.07688 [hep-ph]].

[17] J. Wess and B. Zumino, Phys. Lett. 37B, 95 (1971). doi:10.1016/0370-2693(71)90582-X; E. Witten, Nucl. Phys. B 223, 422 (1983). doi:10.1016/05503213(83)90063-9.

[18] B. I. Abelev et al. [STAR Collaboration], Phys. Rev. Lett. 103, 251601 (2009) doi:10.1103/PhysRevLett.103.251601.

[19] S. A. Voloshin, J. Phys. Conf. Ser. 230, 012021 (2010) doi:10.1088/1742-6596/230/1/012021. 